\title{
Genome-enabled predictions for binomial traits in sugar beet populations
}

\author{
Filippo Biscarini ${ }^{1}{ }^{*}$, Piergiorgio Stevanato ${ }^{2}$, Chiara Broccanello ${ }^{2}$, Alessandra Stella ${ }^{1,3}$ and Massimo Saccomani ${ }^{2}$
}

\begin{abstract}
Background: Genomic information can be used to predict not only continuous but also categorical (e.g. binomial) traits. Several traits of interest in human medicine and agriculture present a discrete distribution of phenotypes (e.g. disease status). Root vigor in sugar beet (B. vulgaris) is an example of binomial trait of agronomic importance. In this paper, a panel of 192 SNPs (single nucleotide polymorphisms) was used to genotype 124 sugar beet individual plants from 18 lines, and to classify them as showing "high" or "low" root vigor.

Results: A threshold model was used to fit the relationship between binomial root vigor and SNP genotypes, through the matrix of genomic relationships between individuals in a genomic BLUP (G-BLUP) approach. From a 5-fold cross-validation scheme, 500 testing subsets were generated. The estimated average cross-validation error rate was $0.000731(0.073 \%)$. Only 9 out of 12326 test observations (500 replicates for an average test set size of 24.65) were misclassified.

Conclusions: The estimated prediction accuracy was quite high. Such accurate predictions may be related to the high estimated heritability for root vigor (0.783) and to the few genes with large effect underlying the trait. Despite the sparse SNP panel, there was sufficient within-scaffold LD where SNPs with large effect on root vigor were located to allow for genome-enabled predictions to work.
\end{abstract}

Keywords: Genomic predictions, Binomial traits, Root vigor, Sugar beet

\section{Background}

Most of current research and applications in genetics are driven by the large quantity of data on individual genomic polymorphisms produced by modern high-throughput genotyping and sequencing technologies [1]. A thriving area is that of genomic predictions in animal and plant science and human medicine.

Genomic data are used to predict future or unobserved events (e.g. disease risk [2]), or the unknown genetic component of given phenotypes (e.g. GEBVs -genomic breeding values- in livestock, crops and trees [3-5]). Such predictions are based on the entire available genomic information, irrespective of the position along the genome or point effects on the response. This ingenious and highly effective "black box" approach was conceived and first

\footnotetext{
*Correspondence: filippo.biscarini@tecnoparco.org

1 Department of Bioinformatics, PTP, Via Einstein - Loc. Cascina Codazza, Lodi, Italy

Full list of author information is available at the end of the article
}

described by Meuwissen et al. around the turn of the millennium [6] and has since then found several applications and started fruitful areas of research.

Genomic information can in principle be used to predict continuous or categorical (ordered or unordered) polygenic traits. Most works so far focussed on continuous traits, while fewer studies dealt with genomic predictions for categorical traits [7-11]. However, several traits of interest in human medicine and agriculture present a discrete distribution of phenotypes (e.g. litter size in mammals), often binomial (e.g. disease status). Statistical methods used for genomic predictions of continuous traits cannot be adequately applied for such traits: the relationship between predictors and binomial phenotypes is logistic rather than linear; the phenotypes follow a binomial rather than normal distribution; the variance is no longer constant but a function of the expectation [12]. Root vigor in sugar beet (B. vulgaris) is an example of binomial trait of agronomic importance. 
In this paper, SNP (single nucleotide polymorphisms) genotypes were used for the classification problem of predicting "high" or "low" root vigor inidividual plants in sugar beet.

Pioneering works on genomic predictions for continuous traits in sugar beet already exist $[13,14]$. However, this is the first study to propose direct modeling of genomic predictions for binomial traits in sugar beet and, to our knowledge, among the few to address this problem in plants in general.

\section{Methods}

\section{Experimental population}

A population of 124 individual sugar beet (B. vulgaris) plants from 18 high- and low-root-vigor lines were available. These lines were characterised by different productivity and were provided by Lion Seeds Ltd. (UK). Root vigor is related to nutrient uptake from the soil and plant productivity [15], and is recorded as a binary trait (either high or low). The lines were phenotyped by measuring the root elongation rate of eleven-days-old seedlings grown under hydroponic conditions. There was no predetermined root elongation rate threshold to classify a sugar beet as having high or low root vigour, and the decision was subjectively made upon phenotypic inspection. The classification has nevertheless been shown to be robust: seedlings classified as "low" or "high" maintain the same class also at the adult plant stage [15]. There were three low-root-vigor (24 individuals) and 15 high-rootvigor (100 individuals) lines. Root elongation rate was $<3$ $\mathrm{mm}$ /day in the low-root-vigor lines and $>6 \mathrm{~mm} /$ day in the high-root-vigor lines.

\section{Marker genotypes and imputation}

All individual plants were genotyped for 192 SNP markers with the high-throughput marker array QuantStudio 12K Flex system coupled with Taqman OpenArray technology. Additional details on the genotyping procedure are described in Stevanato et al., 2013 [16].

The initial genotype screening led to the detection of one duplicated individual (100\% matching genotypes) from a high-root-vigor line, which was removed. The average per-sample and per-marker call-rate was 0.984 and 0.969 . Only one SNP had a per-marker call-rate $\leq 85 \%$ and was removed from the analysis. There were in total 738 missing genotypes (3.14\%). Missing genotypes were imputed based on linkage disequilibrium (LD, [17]). After imputation data were edited for minor allele frequency (MAF): 16 SNPs with MAF $\leq 2.5 \%$ were discarded. This left a total of 123 individuals and 175 SNP markers for the analysis. An overview of the data used in the paper is given in Table 1 . Table 2 reports the distribution of the 175 SNPs (and related scaffolds) used in the analysis
Table 1 Description of the experimental population and SNP marker genotypes

\begin{tabular}{lrr}
\hline N. plant samples & (duplicated) & 124 \\
N. sugarbeet lines & 1 \\
& High-root-vigor lines & 18 \\
& Samples & 15 \\
& Low-root-vigor lines & 100 \\
& Samples & 3 \\
N.SNPs & & 24 \\
Average call-rate & Per SNP & 192 \\
& Per sample & 0.969 \\
Average MAF & 0.984 \\
& N. of SNP call-rate $\leq 85 \%$ & 1 \\
& N. SNPs MAF $\leq 2.5 \%$ & 176 \\
\hline
\end{tabular}

along the 9 chromosomes of the Beta vulgaris genome. The average scaffold size was $1037 \mathrm{kbps}$ (range: 34.5 - 4957 kbps).

\section{Genomic relationships}

Marker genotypes can be used for genome-enabled predictions either by directly estimating and summing their effects over all loci (Ridge Regression BLUP - RR-BLUP) or, indirectly, through the estimation of realized relationships between individuals (genomic BLUP - G-BLUP). These are two different parametrizations of the genomic selection model described in Meuwissen et al. [6]. The two approaches have been shown to be equivalent $[18,19]$.

\begin{tabular}{|c|c|c|}
\hline Chromosome & \# scaffolds & \# SNPs \\
\hline 1 & 6 & 8 \\
\hline 2 & 7 & 11 \\
\hline 3 & 10 & 18 \\
\hline 4 & 18 & 33 \\
\hline 5 & 9 & 16 \\
\hline 6 & 9 & 16 \\
\hline 7 & 14 & 21 \\
\hline 8 & 7 & 10 \\
\hline 9 & 10 & 22 \\
\hline- & 9 & 20 \\
\hline Total & 99 & 175 \\
\hline
\end{tabular}


In this paper SNP genotypes were incorporated in the prediction model through the matrix of genomic relationships between individuals. From imputed genotypes the genomic relationships between individual plants were computed according to Van Raden (2008 [19]) as:

$$
\boldsymbol{G}=\frac{Z \boldsymbol{Z}^{\prime}}{2 \sum p_{i}\left(1-p_{i}\right)}
$$

where $\boldsymbol{G}$ is the matrix of genomic relationships, $Z$ is the matrix of centered SNP genotypes per individual $(-1,0$ or 1 for the homozygous, heterozygous and other homozygous respectively), and $p_{i}$ is the allele frequency at SNP $i$. Genomic relationships were used to model covariances between observations and to evaluate the genetic structure of the population.

\section{Threshold model}

In a GBLUP (Genomic Best Linear Unbiased Predictions) framework [6], the probability $\operatorname{Pr}(Y=[0 / 1] \mid X)$ of having either high or low root vigor given the predictors was modeled assuming a continuous underlying latent variable $l$ ("liability"). A threshold model [12] of the following form was fitted:

$$
l=1 \mu+X g+e
$$

with $\boldsymbol{l}$ the vector of continuous gaussian liabilities, $\boldsymbol{g}$ the vector of additive genetic values of individual plants, and $\boldsymbol{e}$ the vector of logistically distributed residuals; $\boldsymbol{X}$ is a design matrix that allocates records to genetic values. The genetic and residual variances were $\operatorname{Var}(\boldsymbol{g})=\boldsymbol{G} \sigma_{a}^{2}$ and $\operatorname{Var}(\boldsymbol{e})=I \frac{s^{2} \pi^{2}}{3}$ (variance of the logistic distribution, with scale parameter $s=1 ; \boldsymbol{I}$ is the identity matrix). The narrow sense heritability for root vigor was then estimated as:

$$
h^{2}=\frac{\sigma_{a}^{2}}{\sigma_{a}^{2}+\frac{\pi^{2}}{3}}=\frac{\sigma_{a}^{2}}{\sigma_{a}^{2}+3.29}
$$

Low and high root vigor (coded as 0 and 1 respectively) phenotypes were the input of model 2 , which returned a probability (for individuals with known or unknown phenotype) of belonging to either class. The probability of classifying each observation $i$ into high- or low-root-vigor plant was obtained from the cumulative distribution function of the logistic distribution (i.e. the logistic function: $\left.p_{i}=\operatorname{logistic}\left(l_{i}\right)=\frac{e^{\mu+g_{i}}}{1+e^{\mu+g_{i}}}\right)$. Individuals were classified as high-/low-root vigour if $p_{i}>/ \leq 0.5$.

\section{Cross-validation}

In order to obtain a valid estimate of the classification error from model (2), a 5-fold cross validation procedure was adopted [20]. The 123 samples were randomly split into 5 subsets of approximately the same size. In turn, the observations in one subset were set to missing and predicted using the model trained with the remaining four subsets, until all subsets were used once as validation set.
This process was repeated 100 times, each time randomly sampling different subsets, eventually yielding 500 replicates of the analysis. The test error rate in each replicate was computed as:

$$
E R_{(n)}=\frac{1}{n} \sum_{i=1}^{n} E r r_{i}
$$

where $n$ is the number of observations in the test set and $E r r_{i}=I\left(y_{i} \neq \hat{y}_{i}\right)$, with $I(\cdot)$ an indicator function which returns a value of 1 if the predicted and observed phenotypes are different, 0 otherwise. The cross-validation (CV) error rate was then estimated averaging the test error rate over all replicates:

$$
C V_{k}=\frac{1}{k} \sum_{i=1}^{k} E R_{i}
$$

\section{Software}

The programme Beagle was used to impute missing genotypes [17]. The computer package for linear mixed models Asreml was used to fit the threshold model in (2) and estimate variance components [21]. Genomic relationships between plants were estimated with "ad hoc" Python code. Data preparation and figures were produced with the open source statistical environment $R$ [22].

\section{Results}

\section{Realized relationships}

Figure 1 shows the heatmap of estimated genomic relationships between individual plants. Plants have been ordered by root vigor (low and high) and line. Darker colours indicate closer kinship between individuals. The average genomic relationships was 0.365 (standard deviation 0.248; coefficient of variation $67.9 \%$ ). The closest lines appeared to be "LOW1" and "HIGH1" $\left(\overline{a_{i j}}=0.938\right)$; the lines farthest apart were "HIGH2" and "HIGH13" $\left(\overline{a_{i j}}=0.104\right)$.

\section{Heritability and classification}

Model fit was evaluated comparing the full (model (2)) and the reduced (null model: the intercept only) models through a likelihood ratio test. Deviance dropped significantly (p-value $\approx 0$ ), showing good fit of the model. The estimated genetic variance was 11.856 , on the liability scale. From Eq. 3 heritability was then estimated as $h^{2}=0.783$, with a standard error of 0.086 .

From cross-validation, 500 testing subsets were generated. In each of these, observations were classified according to the model fitted to the corresponding training subset, and the classification error calculated as in (4), then averaged over all subsets. The estimated crossvalidation error rate from (5) was 0.000731 (0.073\%). Only 9 out of 12326 test observations (500 replicates for an 


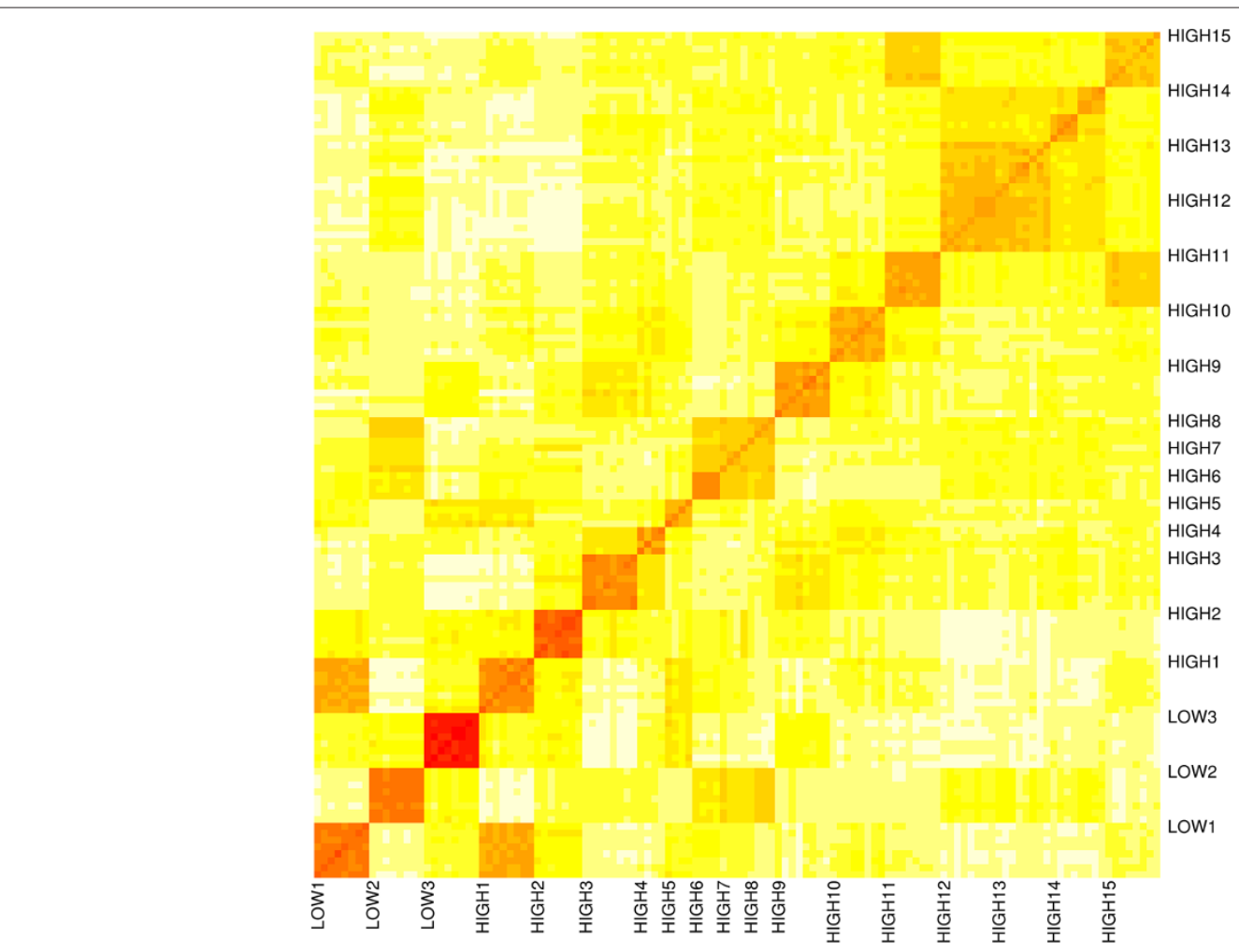

Figure 1 Heatmap of genomic relationships between sugar beet individual plants. Plants have been groupd by line. Darker colors indicate stronger genomic relationships.

average test set size of 24.65) were wrongly classified. All 9 missclassified observations belonged to a single low-root vigor line (line "LOW1").

In the training sets observations were always correctly classified (training error rate $=0$ ). The average estimated probability of having low or high root vigor was calculated for each plant across all 500 replicates of the training and testing sets. The box plots in Figure 2 show the wider probability distribution in the test compared to the training data; the 9 misclassified observations in the test data are represented by points beyond the $P(Y=0 \mid X)=0.5$ dotted threshold line.

\section{Discussion}

In this paper, the problem of classifying binomial phenotypes using SNP markers in sugar beet (B. vulgaris) has been addressed. A very low cross-validation test error rate $(<1 \%)$ was estimated for the genome-based classification of root vigor in sugar beet lines. Genomic predictions with different accuracies have been reported in literature: high (e.g. 0.89 for soluble solids content in apple trees [23]; 0.92 for fat and protein percentage in cattle [24]), moderate (e.g. $\approx 0.60$ for egg weight in laying hens [25]) and low (e.g. 0.38 for stem height in loblolly pines [26]) accuracy of prediction. Wang et al. ([10]) reported accuracies ranging from 0.17 to 0.69 for a simulated categorical trait. In sugar beet, moderate to high prediction accuracies were estimated for a number of traits such as sugar content, molasses loss, root yield and mineral $(\mathrm{Na}, \mathrm{K})$ content $[13,14]$.

The few prediction errors were all observed in line "LOW1". This line had the strongest off-diagonal genomic relationship with line "HIGH1". "LOW1" and "HIGH1" differ for root vigor (and related genes) but share most of their genetic basis. The close relationship of "LOW1" to "HIGH1" (a high root vigor line) may well explain why all 9 misclassifications were observed in this line, considering that SNP genotypes -through the genomic relationship matrix- were used as predictors. The error rate in line "LOW1" was nonetheless very low $(\sim 1.1 \%)$.

The accuracy of genomic predictions is known to depend on a number of factors related to the nature of the analysed trait (e.g. heritability [3]) and to the experimental population at hand (e.g. sample size, number of markers, relatedness between the training and validation sets [3,27]).

Some relevant aspects are discussed below.

\section{Genetic architecture of the trait}

The high predictive ability for root vigor estimated in this study ( $>99 \%$ of correct classifications) may be related 


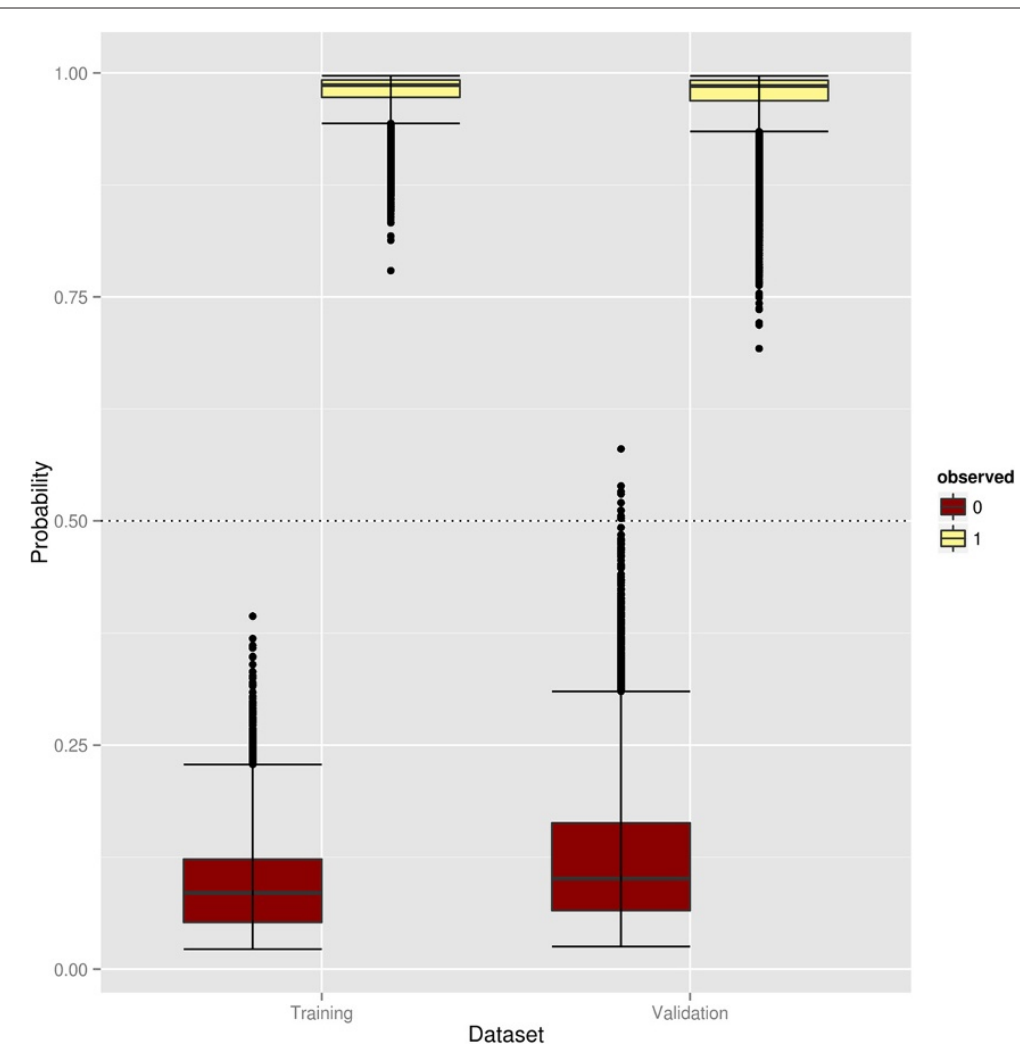

Figure 2 Box plots of estimated probability vs observed root vigor in the training (left) and validation/test (right) data. True high- and low-root-vigor individuals are in light yellow and dark red respectively. The dotted line is the classification threshold $(P(Y=[0 / 1] \mid X)=0.5)$.

to the heritability of the trait and to the number of segregating QTL underlying its expression: this is sometimes referred to as the "genetic architecture" of the trait [28].

Root vigor has been estimated to have high heritability $(\approx 80 \%)$. For a highly heritable trait, using genetic markers to correctly predicting phenotypes is expected to yield good results. The effect of heritability on error rate was checked by fixing the heritability in model (2) instead of estimating it from the data. This has the effect of altering the covariance structure in the GBLUP mixed model equations from model (2) by loosening the relationship between genotypes and phenotypes. Artificially lower heritabilities of $0.5,0.33$ and 0.20 were tested by rerunning the analysis with the same cross-validation scheme and number of replicates. Figure 3 shows the boxplots of the error rate with the true heritability (utmost left column) and with progressively lower heritabilities: both the median error rate and the variance around it incresed when reducing the heritability. The error rate for $h^{2}=0.5$, 0.33 and 0.2 was 0.058 (5.8\%), $0.128(12.8 \%)$ and 0.181 (18.1\%), respectively.

Not only the total genetic variance of a trait plays a role in determining the accuracy of genomic predictions, but also how this variance is spread along the genome (i.e. the distribution of QTL/genes underlying the trait). For a polygenic trait (i.e. determined by a large number of genes) a large number of markers would be needed to ensure that the genetic variance is fully captured. Contrariwise, for an oligogenic trait (i.e. determined only by few genes), even a small number of markers -as in the present study- may be sufficient to capture the genetic variance of the trait, provided that these markers are close to the relevant QTLs. A single-SNP genome-wide association study was performed in order to estimate marker effects for root vigor. A logistic regression model of the form $\operatorname{logit}\left(p_{i}\right)=\mu+S N P_{m}\left(p_{i}=P\left(Y=1 \mid \mu+S N P_{m}\right)\right.$; $S N P_{m}$ : individual genotype at SNP $m$ ) was fit to the data. The magnitude of estimated SNP effects is reported in the barplot in Figure 4. Large marker effects appear to cluster on specific scaffolds from a few chromosomes of the sugar beet genome, while most SNPs do not seem to have an appreciable effect on root vigor. Such distribution of marker effects agrees with an oligogenic basis for the trait root vigor. This, together with the high heritability of the trait, may help explain the very low classification error rate estimated with relatively few markers in this study. 


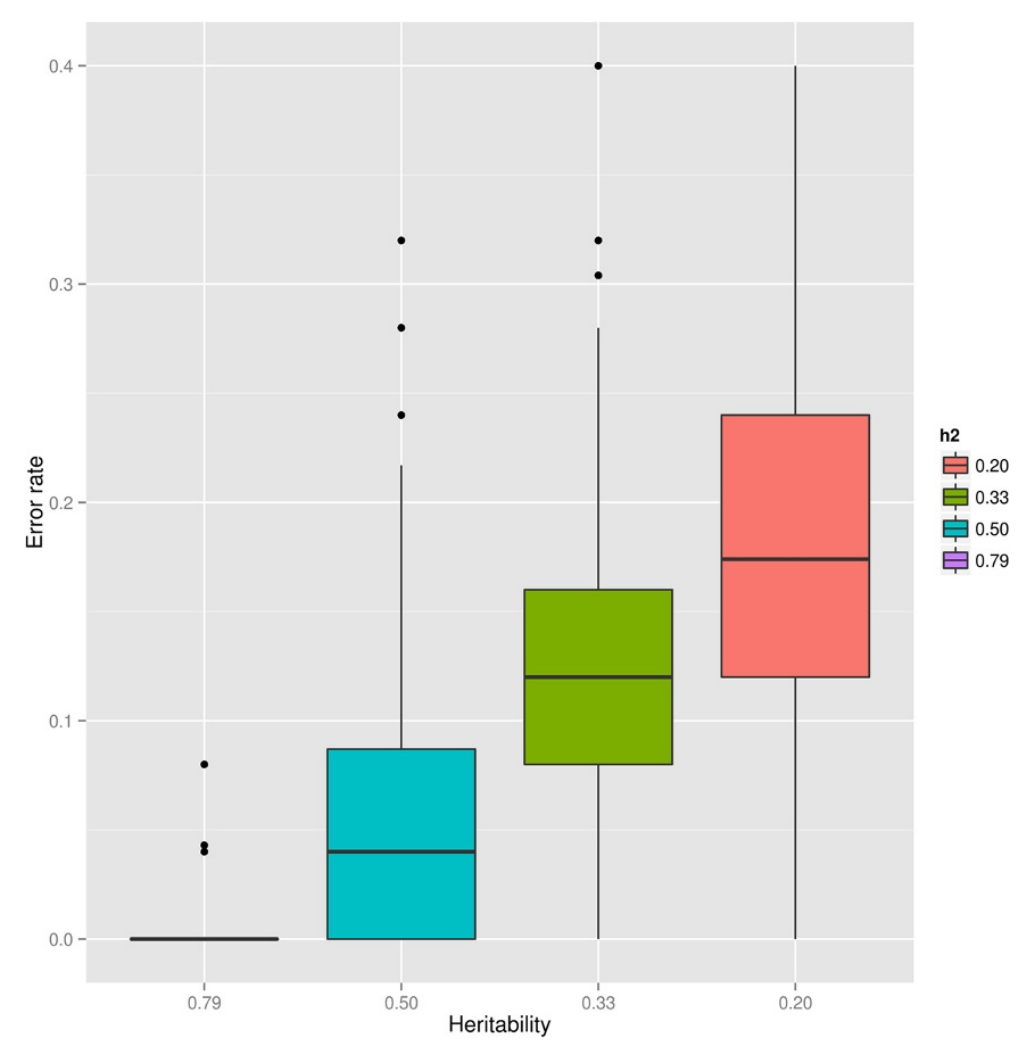

Figure 3 Boxplot of the cross-validation test error rate for the original data $\left(h^{2}=0.783\right)$ and for lower heritabilities.

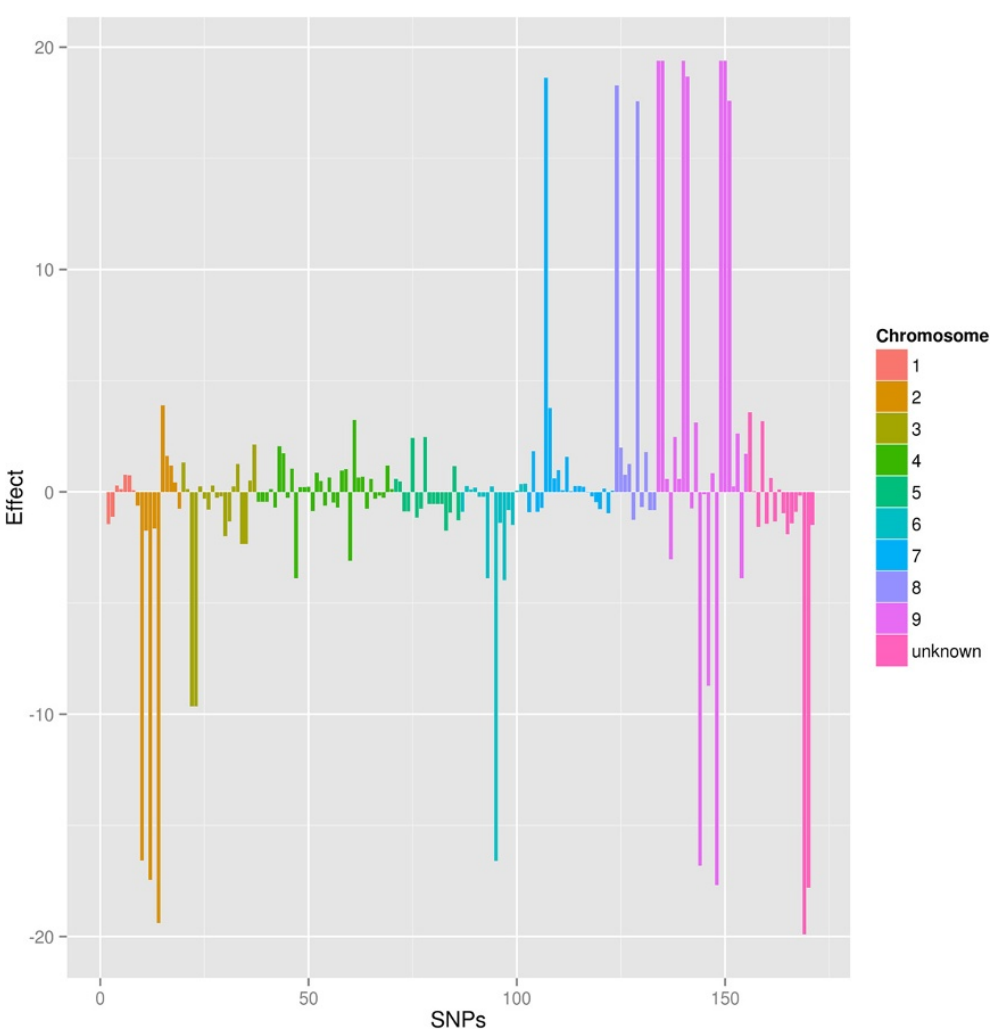

Figure 4 Snp effects for root vigor along the genome of $B$. vulgaris. 


\section{Linkage disequilibrium}

The extent of linkage disequilibrium (LD) in the experimental population is a parameter relevant to the success of genomic predictions. The basic assumption underlying genome-wide predictions is indeed that observed genetic markers and unobserved QTLs are in LD [6]. LD between adjacent markers of around 0.2 -measured as $r^{2}$ [29]- is deemed to be required for reliable genomic predictions [3]. Dense marker panels ensure that there is sufficient LD between markers. With sparser panels this may not be the case. The available release of the $B$. vulgaris genome was not assembled in chromosomes, but organised in 82305 scaffolds (and contigs). The sugar beet genome sequence comprises $567 \mathrm{Mbps}$ of which $85 \%$ could be assigned to chromosomes [30]. Most scaffolds -but not all- could therefore be mapped to chromosomes; however, the relative position of the scaffolds along the chromosomes was not known. Therefore, pairwise LD between adjacent SNPs could be estimated only within scaffold.

The total average estimated LD between all pairs of markers, measured as $r^{2}$, was 0.061 . This values is below what is needed for genomic predictions to work, but refers to all markers, not only adjacent markers. Adjacent markers could be determined only within-scaffold; the average within-scaffold pairwise LD was $r^{2}=0.404$, which seems to be largely sufficient for reliable genomic predictions. Also the LD between markers with large effect on root vigor (see Figure 4) was estimated: this was $r^{2}=0.327$ on average, and can be interpreted as an indirect estimate of the LD between markers and QTLs.

Figure 5 reports the LD heatmap between all markers (large panel on the left) and between markers on three scaffolds (small panels on the right): though no clear LD patterns emerge from the total set of SNPs, a strong LD structure is present on individual scaffolds.

\section{Imputation accuracy}

Imputing missing genotypes is usually a preliminary step to the analysis of genomic data. After markers and individuals with low call-rate are edited out, there is usually still a small proportion of uncalled genotypes (e.g. $<5 \%$ ) randomly distributed along the genome. Such missing genotypes are imputed using pedigree-based or pedigreefree methods. Imputation accuracy is typically very high; for instance, $>95 \%$ correctly imputed genotypes were reported in maize [31] and cattle [32]. This usually applies to scenarios in which moderate to high density marker panels are available. With fewer markers genotype imputation may be less accurate, as a consequence of lower LD. This may be especially true for pedigree-free imputation methods, which rely heavily on between marker LD.

In order to estimate the accuracy with which genotypes were imputed in the present study, a subset with no missing genotypes was extracted from the total dataset. Increasing proportions of missing data were then artificially introduced in the data: $1 \%, 2 \%, 3 \%, 5 \%, 10 \%$ and $20 \%$.

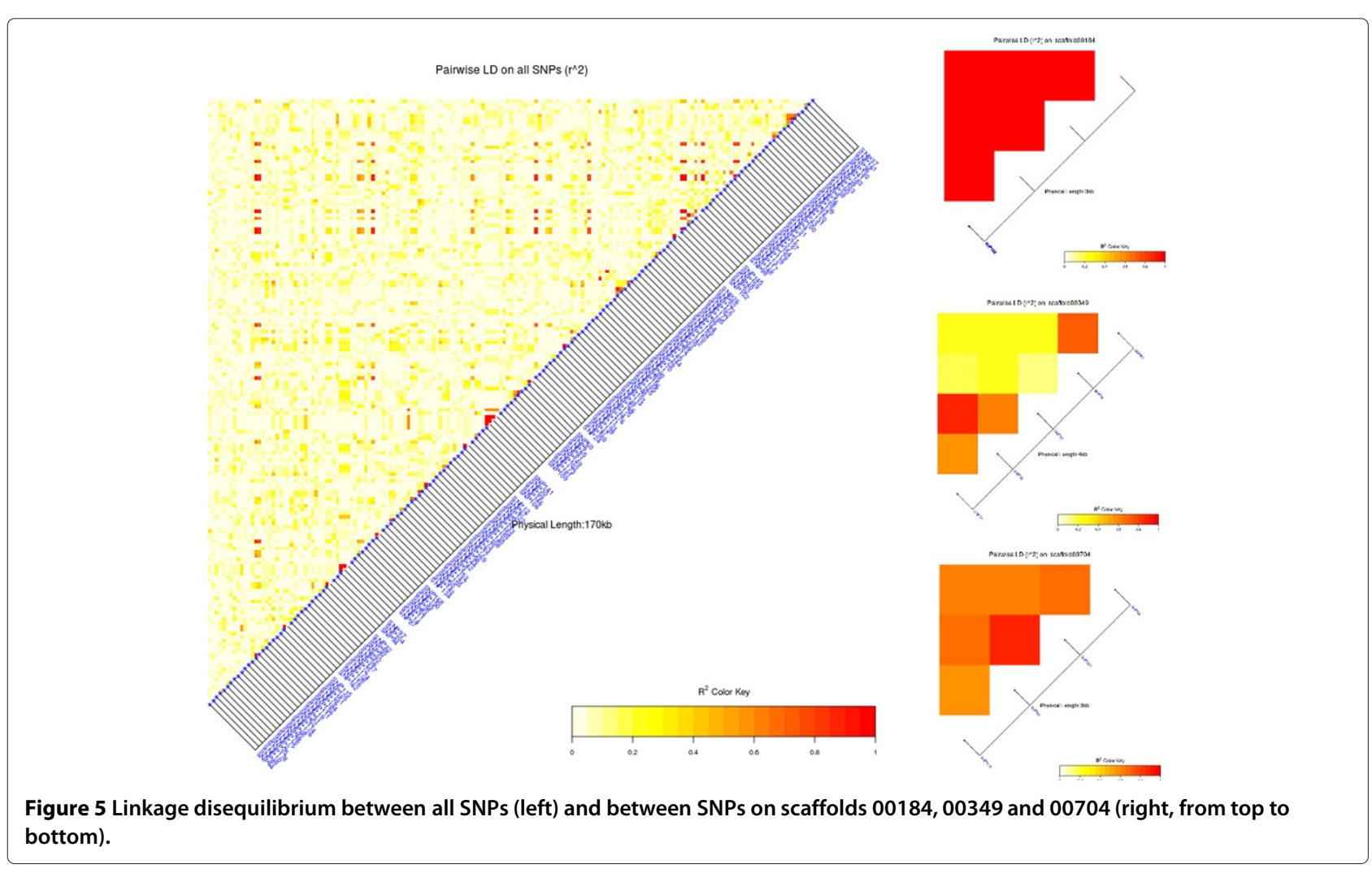


Table 3 Imputation accuracy with increasing proportions of missing genotypes

\begin{tabular}{ccccccc}
\hline \% missing & $\mathbf{1 \%}$ & $\mathbf{2 \%}$ & $\mathbf{3 \%}$ & $\mathbf{5 \%}$ & $\mathbf{1 0 \%}$ & $\mathbf{2 0 \%}$ \\
\hline accûracy & 0.8405 & 0.8402 & 0.8402 & 0.8396 & 0.8301 & 0.8089
\end{tabular}

The average proportion (over 5 replicates) of correctly imputed genotypes (accûracy) was used to estimate imputation accuracy.

For each proportion of missing genotypes, 5 random replicates were generated. The average proportion of correctly imputed genotypes over 5 replicates for each proportion of missing data was then used to estimate an empirical curve of the imputation accuracy. Results are summarised in Table 3: the intersection between the empirical curve and the percentage of missing genotypes in the original dataset (3.14\%), provided an indirect estimate of the imputation accuracy obtained in this study: 0.840. This estimate appears to be quite robust, considering that up to $10 \%$ missing genotypes the empirical imputation accuracy curve is substantially flat, and only for missing data $>10 \%$ the accuracy of imputation seems to drop. An imputation error of about $16 \%$ is higher than what is typically found in humans and commercial crop and livestock populations. This may be due to the lower extent of LD estimated in this population with the availabe SNP panel, and to the lack of a mature assembly of the genome (partial information on chromosome structure and marker postion).

\section{Comparison with another classification method}

The threshold model used in this study for genomeenabled prediction of the binary trait root vigor in sugar beet was compared with Support Vector Machine (SVM), another widely adopted method for classification of categorical observations [20,33].

The kernel function and tuning parameter $C$ to be used in SVM were chosen so to minimize the classification error through 5-fold cross-validation. A linear kernel $\left(K\left(x_{i}, x_{i^{\prime}}\right)=\left\langle x_{i}, x_{i^{\prime}}\right\rangle=\sum_{j=1}^{p} x_{i j} x_{i^{\prime} j}\right.$, for individual plant $i$ and $p$ parameters) and $C=0.01$ were chosen and used to classify sugar beet individual plants with SVM in the same cross-validation procedure adopted for the threshold model (5-fold, 100 repetitions). The estimated error rate was close to zero $(0.025 \%)$, in line with what was obtained with the threshold model $(0.073 \%)$. The two classifiers were compared also by looking at the ROC curves [34]: the two curves overlapped almost completely, having both an area under the curve (AUC) close to $1(\sim 0.98)$. This shows that with both classifiers the total error rate and the number of false positives and false negatives were very low.

\section{Applications to sugar beet breeding}

Root vigor, expressed as high root elongation rate, is essential for the efficient acquisition of mobile soil nutrients
[35]; this is especially true in presence of water-nutritional stress [36]. The increased root elongation rate in response to low water availability or nutrient deprivation allow plants to circumvent water or nutrients limitations [37]. Of all sugar beet morphological root traits, root elongation rate shows the largest variation between high- and low-yielding genotypes and was shown to be significantly correlated with sugar beet yield [15].

Root traits are difficult to be measured accurately and this is an obstacle to reliable and effective selection. Genomic data can be used for early and accurate prediction of root vigor in sugar beet seeds, thereby enhancing the efficiency of breeding for rhizospheric stress tolerance and yield in sugar beet. Improvements are likely to come from shortened breeding cycles and more accurate and less expensive phenotypic evaluation.

\section{Conclusions}

In this paper, the use of genomic information to predict a binomially distributed phenotype (root vigor) in sugar beet populations was presented. Prediction accuracy proved to be quite high, with an estimated crossvalidation error rate close to zero (0.073\%). Such excellent prediction performance may be related to properties of the analysed trait and available population. Root vigor was estimated to have high heritability $(0.783)$ and to be determined by few genes with large effect. Despite the sparse SNP panel, there was sufficient within-scaffold LD where SNPs with large effect on root vigor were located. For an oligogenic highly heritable trait with a favorable distribution of markers on the genome, even with relatively few SNPs very accurate predictions can be achieved. The results described in this paper constitute an interesting application of genomic predictions to binomial (and more generally categorical/multinomial) traits, and may lead to promising applications of genomic selection in sugar beet breeding programmes.

\section{Competing interests \\ The authors declare that they have no competing interests.}

\section{Authors' contributions}

FB carried out all statistical analyses and drafted most of the manuscript. PS, CB and MS selected the experimental population and generated all molecular and phenotypic data. AS contributed ideas to the work and drafted parts of the manuscript. All authors read and approved the final manuscript.

\section{Acknowledgements}

This research was financially supported by the Marie Curie European Reintegration Grant "NEUTRADAPT".

\section{Author details}

1 Department of Bioinformatics, PTP, Via Einstein - Loc. Cascina Codazza, Lodi, Italy. ${ }^{2}$ DAFNE, Università di Padova, 24105 Padova, Italy. ${ }^{3}$ IBBA-CNR, Via Einstein, 26900 Lodi, Italy.

Received: 7 April 2014 Accepted: 4 July 2014

Published: 22 July 2014 
References

1. Van Tassell CP, Smith TP, Matukumalli LK, Taylor JF, Schnabel RD, Lawley $C T$, Haudenschild CD, Moore SS, Warren WC, Sonstegard TS: Snp discovery and allele frequency estimation by deep sequencing of reduced representation libraries. Nat Med 2008, 5(3):247-252.

2. Abraham G, Tye-Din JA, Bhalala OG, Kowalczyk A, Zobel J, Inouye M: Accurate and robust genomic prediction of celiac disease using statistical learning. PloS one 2013, 10(2):1004137.

3. Hayes B, Bowman P, Chamberlain A, Goddard M: Invited review: genomic selection in dairy cattle: Progress and challenges. $J$ Dairy S $\mathrm{Ci}$ 2009, 92(2):433-443.

4. Albrecht T, Wimmer V, Auinger H-J, Erbe M, Knaak C, Ouzunova M Simianer H, Schön C-C: Genome-based prediction of testcross values in maize. Theor Appl Genet 2011, 123(2):339-350.

5. Kumar S, Bink MC, Volz RK, Bus VG, Chagné D: Towards genomic selection in apple (malus $x$ domestica borkh.) breeding programmes: prospects, challenges and strategies. Tree Genet Genomes 2012, 8(1):1-14.

6. Meuwissen T, Hayes B, Goddard ME: Prediction of total genetic value using genome-wide dense marker maps. Genetics 2001, 157(4):1819-1829.

7. González-Recio O, Gianola D, Long N, Weigel KA, Rosa GJ, Avendaño S: Nonparametric methods for incorporating genomic information into genetic evaluations: an application to mortality in broilers. Genetics 2008, 178(4):2305-2313.

8. Villanueva B, Fernández J, García-Cortés L, Varona L, Daetwyler H, Toro M: Accuracy of genome-wide evaluation for disease resistance in aquaculture breeding programs. J Anim Sci 2011, 89(11):3433-3442.

9. Manor $\mathrm{O}$, Segal E: Predicting disease risk using bootstrap ranking and classification algorithms. PLOS Comput Biol 2013, 9(8):1003200

10. Wang C, Ding X, Wang J, Liu J, Fu W, Zhang Z, Yin Z, Zhang Q: Bayesian methods for estimating gebvs of threshold traits. Heredity 2013, 110(3):213-219.

11. Ornella L, Pérez P, Tapia E, González-Camacho J, Burgueño J, Zhang X, Singh S, Vicente F, Bonnett D, Dreisigacker S, Singh R, Long N, Crossa J: Genomic-enabled prediction with classification algorithms. Heredity 2014, 112(6):616-626.

12. Gianola D: Theory and analysis of threshold characters. J Anim Sci 1982, 54(5):1079-1096.

13. Hofheinz N, Borchardt D, Weissleder K, Frisch M: Genome-based prediction of test cross performance in two subsequent breeding cycles. Theor App/ Genet 2012, 125(8):1639-1645.

14. Würschum T, Reif JC, Kraft T, Janssen G, Zhao Y: Genomic selection in sugar beet breeding populations. BMC Genet 2013, 14(1):85.

15. Stevanato $P$, Trebbi $D$, Saccomani M: Root traits and yield in sugar beet: identification of aflp markers associated with root elongation rate. Euphytica 2010, 173(3):289-298.

16. Stevanato P, Broccanello C, Biscarini F, Del Corvo M, Sablok G, Panella L, Stella A, Concheri G: High-throughput rad-snp genotyping for characterization of sugar beet genotypes. Plant Mol Biol Rep 2014, 32(3):691-696.

17. Browning SR, Browning BL: Rapid and accurate haplotype phasing and missing-data inference for whole-genome association studies by use of localized haplotype clustering. Am J Hum Genet 2007 81(5):1084-1097.

18. Habier D, Fernando R, Dekkers J: The impact of genetic relationship information on genome-assisted breeding values. Genetics 2007. 177(4):2389-2397.

19. VanRaden P: Efficient methods to compute genomic predictions. Dairy Sci 2008, 91(11):4414-4423.

20. Hastie T, Tibshirani R, Friedman J: Model assessment and selection. In The Elements of Statistical Learning. Volume 2. New York, NY, USA: Springer New York Inc.; 2009:241-247.

21. Gilmour AR, Gogel B, Cullis B, Thompson R: Asrem/ User Guide Release 3.0. Hemel Hempstead: VSN International Ltd; 2009

22. R Development Core Team: R: A Language and Environment for Statistical Computing. Vienna: R Foundation for Statistical Computing; 2008. ISBN 3-900051-07-0. [http://www.R-project.org]

23. Kumar S, Chagné D, Bink MC, Volz RK, Whitworth C, Carlisle C: Genomic selection for fruit quality traits in apple (malus $\mathrm{x}$ domestica borkh.). PloS one 2012, 7(5):36674.
24. Charfeddine N, Rodríquez-Ramilo ST, Jiménez-Montero JA, Carabaño MJ, González-Recio O: Non parametric vs. gblup model for genomic evaluation with large reference population in holstein cattle. Interbull Bull 2013, 47:67-76.

25. Wolc A, Stricker C, Arango J, Settar P, Fulton JE, O'Sullivan NP, Preisinger R, Habier D, Fernando R, Garrick DJ, Lamont SJ, Dekkers JCM: Breeding value prediction for production traits in layer chickens using pedigree or genomic relationships in a reduced animal model. Genet Sel Evol 2011, 43(5):1-9.

26. Resende MF, Muñoz P, Resende MD, Garrick DJ, Fernando RL, Davis JM, Jokela EJ, Martin TA, Peter GF, Kirst M: Accuracy of genomic selection methods in a standard data set of loblolly pine (pinus taeda I.). Genetics 2012, 190(4):1503-1510.

27. Pszczola M, Strabel T, Mulder $H$, Calus M: Reliability of direct genomic values for animals with different relationships within and to the reference population. J Dairy Sci 2012, 95(1):389-400.

28. Daetwyler HD, Pong-Wong R, Villanueva B, Woolliams JA: The impact of genetic architecture on genome-wide evaluation methods. Genetics 2010, 185(3):1021-1031

29. Hill W, Robertson A: Linkage disequilibrium in finite populations. Theor App/ Genet 1968, 38(6):226-231.

30. Dohm JC, Minoche AE, Holtgräwe D, Capella-Gutiérrez S, Zakrzewski F, Tafer H, Rupp O, Sörensen TR, Stracke R, Reinhardt R, Goesmann A, Kraft T, Schulz B, Stadler PF, Schmidt T, Gabaldón T, Lehrach H, Weisshaar B, Himmelbauer $\mathrm{H}$ : The genome of the recently domesticated crop plant sugar beet (beta vulgaris). Nature 2013, 505:546-549.

31. Hickey JM, Crossa J, Babu R, de los Campos G: Factors affecting the accuracy of genotype imputation in populations from several maize breeding programs. Crop Sci 2012, 52(2):654-663.

32. VanRaden PM, O'Connell JR, Wiggans GR, Weigel KA: Genomic evaluations with many more genotypes. Genet Sel Evol 2011, 43:10.

33. Hornik K, Meyer D, Karatzoglou A: Support vector machines in r. J Stat Software 2006, 15(9):1-28.

34. Fawcett T: Roc graphs: Notes and practical considerations for researchers. Mach Learn 2004, 31:1-38.

35. Stevanato P, Saccomani M, Bertaggia M, Bottacin A, Cagnin M, De Biagg M, Biancardi E: Nutrient uptake traits related to sugarbeet yield. $J$ Sugar Beet Res 2004, 41:89-100.

36. Saccomani M, Stevanato P, Trebbi D, McGrath JM, Biancardi E: Molecular and morpho-physiological characterization of sea, ruderal and cultivated beets. Euphytica 2009, 169(1):19-29.

37. Huang B: Water relations and root activities of buchloe dactyloides and zoysia japonica in response to localized soil drying. Plant Soil 1999, 208(2):179-186.

doi:10.1186/1471-2156-15-87

Cite this article as: Biscarini et al:: Genome-enabled predictions for binomial traits in sugar beet populations. BMC Genetics 2014 15:87.

\section{Submit your next manuscript to BioMed Central} and take full advantage of:

- Convenient online submission

- Thorough peer review

- No space constraints or color figure charges

- Immediate publication on acceptance

- Inclusion in PubMed, CAS, Scopus and Google Scholar

- Research which is freely available for redistribution 